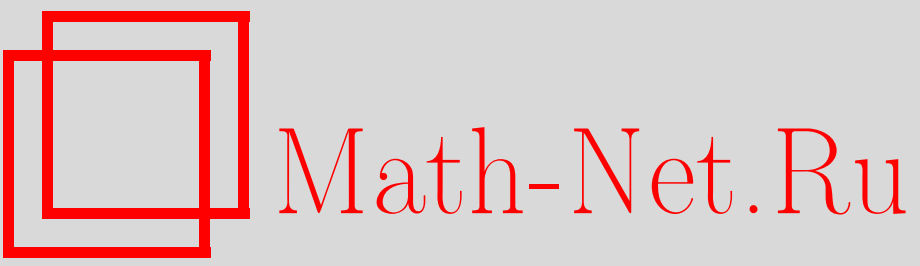

О. А. Репин, С. К. Кумыкова, Нелокальная задача для уравнения смешанного типа третьего порядка с обобщенными операторами дробного интегродифференцирования произвольного порядка, Вестн. Сам. гос. техн. ун-та. Сер. Физ.-мат. науки, 2011, выпуск 4(), 25-36

DOI: https://doi.org/10.14498/vsgtu1014

Использование Общероссийского математического портала Math-Net.Ru подразумевает, что вы прочитали и согласны с пользовательским соглашением http: //www. mathnet.ru/rus/agreement

Параметры загрузки:

IP : 54.162 .27 .143

26 апреля 2023 г., 17:24:09 
УДК 517.956.6 + 517.968.23

\section{НЕЛОКАЛЬНАЯ ЗАДАЧА ДЛЯ УРАВНЕНИЯ СМЕШАННОГО ТИПА ТРЕТЬЕГО ПОРЯДКА С ОБОБЩЁННЫМИ ОПЕРАТОРАМИ ДРОБНОГО ИНТЕГРО-ДИФФЕРЕНЦИРОВАНИЯ ПРОИЗВОЛЬНОГО ПОРЯДКА}

\section{О. А. Репин ${ }^{1}$, С.К. Кумъкова ${ }^{2}$}

1 Самарский государственный экономический университет,

443090, Самара, ул. Советской Армии, 141.

2 Кабардино-Балкарский государственный университет им. Х. М. Бербекова, 360004, Нальчик, ул. Чернышевского, 173.

E-mails: matstat@mail.ru, bsk@rect.kbsu.ru

Исследована однозначная разрешимость внутреннекраевой задачи для уравнения смешанного типа третьего порядка с кратными характеристиками. При ограничениях неравенственного вида на известные бункиии и различных порядках операторов дробного интегро-дифберенцирования доказана теорема единственности. Существование решения задачи эквивалентно редуцировано к вопросу разрешимости интегрального уравнения Фредгольма второго рода.

Ключевые слова: краевая задача, операторы дробного интегро-дифберенцирования, гипергеометрическая функиия Гаусса, интегральное уравнение Фредгольма.

Введение. Рассмотрим уравнение

$$
0=\left\{\begin{aligned}
u_{x x x}+a_{1}(x, y) u_{x}+a_{0}(x, y) u-b(x, y) u_{y}, & y>0 \\
(-y)^{m} u_{x x}-u_{y y}+a(-y)^{\frac{m}{2}-1} u_{x}, & y<0
\end{aligned}\right.
$$

(здесь $a$-вещественная постоянная, $m>2$ ) в конечной области $\Omega$, ограниченной отрезками $A A_{0}, B B_{0}, A_{0} B_{0}$ прямых $x=0, x=1, y=1$ соответственно, лежащих в полуплоскости $y>0$, и его характеристиками

$$
A C: x-\frac{2}{m+2}(-y)^{\frac{m+2}{2}}=0, \quad B C: x+\frac{2}{m+2}(-y)^{\frac{m+2}{2}}=1
$$

в полуплоскости $y<0$.

Пусть $\Omega_{1}=\Omega \cap(y>0), \Omega_{2}=\Omega \cap(y<0), I \equiv A B$ - единичный интервал $0<x<1$ прямой $y=0$.

ЗАДАчА. Найти функцию $u(x, y) \in C(\bar{\Omega}) \cap C^{1}(\Omega) \cap C_{x, y}^{(3,1)}\left(\Omega_{1}\right) \cap C_{x, y}^{(2,2)}\left(\Omega_{2}\right)$, $u_{x} \in C\left(\bar{\Omega}_{1}\right)$, являюшууюся решением уравнения (1) в $\Omega$ при $y \neq 0$ и удовлетворяющую условиям

$$
\begin{gathered}
u(0, y)=\varphi_{1}(y), \quad u(1, y)=\varphi_{2}(y), \quad 0 \leqslant y \leqslant 1 \\
u_{x}(0, y)-u_{x}(1, y)=\varphi_{3}(y), \quad 0 \leqslant y \leqslant 1 \\
A(x)\left(I_{0+}^{\alpha_{1}, \beta_{1}, \eta_{1}} u\left[\Theta_{0}(t)\right]\right)(x)+B(x)\left(I_{1-}^{\alpha_{2}, \beta_{2}, \eta_{2}} u\left[\Theta_{1}(t)\right]\right)(x)+ \\
+C(x) u_{y}(x, 0)+D(x) u(x, 0)=\gamma(x) \quad \forall x \in I,
\end{gathered}
$$

Олег Александрович Репин (д.ф.-м.н., проф.), зав. кафедрой, каф. математической статистики и эконометрики. Светлана Каншубиевна Кумыкова (к.ф.-м.н.), доцент, каф. теории функций и функционального анализа. 
где $\varphi_{i}(y)(i=1,2,3), A(x), B(x), C(x), D(x), \gamma(x)$ - заданные функиии, такue, что $A^{2}(x)+B^{2}(x)+C^{2}(x)+D^{2}(x) \neq 0, A(x), B(x), C(x), D(x), \gamma(x) \in$ $C^{1}(\bar{I}) \cap C^{3}(I), a_{0}(x, y) \in C\left(\bar{\Omega}_{1}\right), a_{1}(x, y), b(x, y) \in C^{2}\left(\bar{\Omega}_{1}\right) ; \Theta_{0}(x)$ u $\Theta_{1}(x)-$ moчки пересечения характеристик уравнения (1), выходящих из точки $(x, 0) \in I$, с характеристиками АC и ВC соответственно; $\left(I_{0+}^{\alpha, \beta, \eta} f\right)(x) u\left(I_{1-}^{\alpha, \beta, \eta} f\right)(x)-$ обобщённые операторы дробного интегро-дифферениирования с гипергеометрической фучнкией Гаусса $F(a, b ; c ; z)$, введённъе в работе [1] (cм. также [2, с. 326-327], [3, с. 14]), и имеююие при действительных $\alpha, \beta, \eta$ и $x>0$ следующие записи:

$$
\begin{gathered}
\left(I_{0+}^{\alpha, \beta, \eta} f\right)(x)=\frac{x^{-\alpha-\beta}}{\Gamma(\alpha)} \int_{0}^{x}(x-t)^{\alpha-1} F\left(\alpha+\beta,-\eta ; \alpha ; 1-\frac{t}{x}\right) f(t) d t \quad(\alpha>0), \\
\left(I_{0+}^{\alpha, \beta, \eta} f\right)(x)=\left(\frac{d}{d x}\right)^{n}\left(I_{0+}^{\alpha+n, \beta-n, \eta-n} f\right)(x) \quad(\alpha \leqslant 0, n=[-\alpha]+1) ; \\
\left(I_{1-}^{\alpha, \beta, \eta} f\right)(x)=\frac{(1-x)^{-\alpha-\beta}}{\Gamma(\alpha)} \int_{x}^{1}(t-x)^{\alpha-1} F\left(\alpha+\beta,-\eta ; \alpha ; \frac{t-x}{1-x}\right) f(t) d t(\alpha>0), \\
\left(I_{1-}^{\alpha, \beta, \eta} f\right)(x)=\left(-\frac{d}{d x}\right)^{n}\left(I_{1-}^{\alpha+n, \beta-n, \eta-n} f\right)(x) \quad(\alpha \leqslant 0, n=[-\alpha]+1),
\end{gathered}
$$

в частности

$$
\left(I_{0+}^{0,0, \eta} f\right)(x)=f(x), \quad\left(I_{1-}^{0,0, \eta} f\right)(x)=f(x) .
$$

Для уравнения третьего порядка с кратными характеристиками в работах $[4,5]$ исследовались нелокальные задачи с операторами дробного в смысле Римана дифференцирования.

Данная работа обобщает полученные ранее результаты и является продолжением этих исследований.

\section{1. Единственность решения задачи.}

Теорема. В области $\Omega$ не может существовать более одного решения задачи (1)-(4), если

$$
2 a_{0}(x, y)-a_{1_{x}}(x, y)+b_{y}(x, y)-2 b(x, y) N>0 \quad \text { в области } \Omega_{1},
$$

где $N=$ const, удовлетворлющая условию

$$
\begin{gathered}
N>\max _{\bar{\Omega}_{1}} \frac{2 a_{0}-\frac{\partial a_{1}}{\partial x}+\frac{\partial b}{\partial y}}{2 b}, \\
b(x, y)>\rho>0, \quad b(x, 0)=\mathrm{const}, \\
2 a_{0}(x, 0)-a_{1}^{\prime}(x, 0) \leqslant 0,
\end{gathered}
$$

а также либо

$$
\alpha_{1}=-\alpha, \quad \beta_{1}=0, \quad \alpha_{2}=-\beta, \quad \beta_{2}=0
$$

и выполняются условия

$$
E_{1}(x)=\frac{\Gamma(\alpha+\beta)}{\Gamma(\beta)} A(x)+\frac{\Gamma(\alpha+\beta)}{\Gamma(\alpha)} B(x)+D(x) \neq 0 \quad \forall x \in \bar{I},
$$




$$
\left[\frac{A(x)}{E_{1}(x)}\right]^{\prime} \geqslant 0, \quad\left[\frac{B(x)}{E_{1}(x)}\right]^{\prime} \leqslant 0, \quad \frac{C(x)}{E_{1}(x)} \geqslant 0 \quad \forall x \in \bar{I},
$$

либо

$$
\alpha_{1}=\beta-1, \quad \beta_{1}=1-\alpha-\beta, \quad \alpha_{2}=\alpha-1, \quad \beta_{2}=1-\alpha-\beta
$$

и выполняются условия

$$
\begin{gathered}
E_{2}(x)=\Gamma(2-\alpha-\beta)\left(\frac{m+2}{4}\right)^{\frac{2}{m+2}}\left[\frac{A(x)}{\Gamma(1-\alpha)}+\frac{B(x)}{\Gamma(1-\beta)}\right]+C(x) \neq 0 \quad \forall x \in \bar{I}, \\
{\left[\frac{A(x)}{E_{2}(x)}\right]^{\prime} \geqslant 0, \quad\left[\frac{B(x)}{E_{2}(x)}\right]^{\prime} \leqslant 0, \quad \frac{D(x)}{E_{2}(x)} \geqslant 0 \quad \forall x \in \bar{I},}
\end{gathered}
$$

причём $A(1)=0, B(0)=0 ; \alpha=\frac{m-2 a}{2(m+2)}, \beta=\frac{m+2 a}{2(m+2)}$.

Доказ а тель ст в о. Устремляя $y \rightarrow+0$ в уравнении (1), получим функциональное соотношение между $\tau(x)=u(x, 0)$ и $v(x)=u_{y}(x, 0)$, принесённое на отрезок $\bar{I}$ из области $\Omega_{1}$ :

$$
v(x)=\bar{\alpha}(x) \tau^{\prime \prime \prime}(x)+\bar{\beta}(x) \tau^{\prime}(x)+\bar{\gamma}(x) \tau(x),
$$

где $\bar{\alpha}(x)=1 / b(x, 0), \bar{\beta}(x)=a_{1}(x, 0) / b(x, 0), \bar{\gamma}(x)=a_{0}(x, 0) / b(x, 0)$.

Рассмотрим интеграл $I^{*}=\int_{0}^{1} \tau(x) v(x) d x$. Подставляя $v(x)$ из $(14)$, с учётом (2), (3) будем иметь [5]

$$
\begin{aligned}
I^{*}=\frac{1}{2}[\bar{\alpha}(0)-\bar{\alpha}(1)]\left(\tau^{\prime}(0)\right)^{2}+ & \frac{3}{2} \int_{0}^{1} \bar{\alpha}^{\prime}(x)\left(\tau^{\prime}(x)\right)^{2} d x+ \\
& +\int_{0}^{1}\left[\bar{\gamma}(x)-\frac{1}{2} \bar{\beta}^{\prime}(x)-\frac{1}{2} \bar{\alpha}^{\prime \prime \prime}(x)\right] \tau^{2}(x) d x .
\end{aligned}
$$

Из $(15)$ в силу условий $(6),(7)$ легко заключить, что $I^{*} \leqslant 0$.

Используя формулу решения задачи Коши для уравнения (1) в области $\Omega_{2}[6]$, можно записать

$$
\begin{aligned}
& u\left[\Theta_{0}(x)\right]=\frac{\Gamma(\alpha+\beta)}{\Gamma(\beta)}\left(I_{0+}^{\alpha, 0, \beta-1} \tau(t)\right)(x)+ \\
& \quad+\frac{\Gamma(2-\alpha-\beta)}{\Gamma(1-\alpha)}\left(\frac{m+2}{4}\right)^{\frac{2}{m+2}}\left(I_{0+}^{1-\beta, \alpha+\beta-1, \beta-1} v(t)\right)(x), \\
& u\left[\Theta_{1}(x)\right]=\frac{\Gamma(\alpha+\beta)}{\Gamma(\alpha)}\left(I_{1-}^{\beta, 0, \alpha-1} \tau(t)\right)(x)+ \\
& \quad+\frac{\Gamma(2-\alpha-\beta)}{\Gamma(1-\beta)}\left(\frac{m+2}{4}\right)^{\frac{2}{m+2}}\left(I_{1-}^{1-\alpha, \alpha+\beta-1, \alpha-1} v(t)\right)(x) .
\end{aligned}
$$


Подставляя $u\left[\Theta_{0}(x)\right]$ и $u\left[\Theta_{1}(x)\right]$ в краевое условие (4), опираясь на формулы композиций при $\gamma>0$ (см., например, [2, с. 327])

$$
\left(I_{0+}^{\alpha, \beta, \eta} I_{0+}^{\gamma, \delta, \alpha+\eta} f\right)(x)=\left(I_{0+}^{\alpha+\gamma, \beta+\delta, \eta} f\right)(x),\left(I_{1-}^{\alpha, \beta, \eta} I_{1-}^{\gamma, \delta, \alpha+\eta} f\right)(x)=\left(I_{1-}^{\alpha+\gamma, \beta+\delta, \eta} f\right)(x),
$$

получим

$$
\begin{aligned}
& \frac{\Gamma(\alpha+\beta)}{\Gamma(\beta)} A(x)\left(I_{0+}^{\alpha_{1}+\alpha, \beta_{1}, \beta-1-\alpha_{1}} \tau(t)\right)(x)+ \\
& \quad+\frac{\Gamma(\alpha+\beta)}{\Gamma(\alpha)} B(x)\left(I_{1-}^{\alpha_{2}+\beta, \beta_{2}, \alpha-1-\alpha_{2}} \tau(t)\right)(x)+ \\
& +\frac{\Gamma(2-\alpha-\beta)}{\Gamma(1-\alpha)}\left(\frac{m+2}{4}\right)^{\frac{2}{m+2}} A(x)\left(I_{0+}^{\alpha_{1}+1-\beta, \beta_{1}+\alpha+\beta-1, \beta-1-\alpha_{1}} v(t)\right)(x)+ \\
& +\frac{\Gamma(2-\alpha-\beta)}{\Gamma(1-\beta)}\left(\frac{m+2}{4}\right)^{\frac{2}{m+2}} B(x)\left(I_{1-}^{\alpha_{2}+1-\alpha, \beta_{2}+\alpha+\beta-1, \alpha-1-\alpha_{2}} v(t)\right)(x)+ \\
& \quad+C(x) v(x)+D(x) \tau(x)=\gamma(x) .
\end{aligned}
$$

Пусть выполняются условия (11), (12) теоремы. Тогда при $\gamma(x)=0$ соотношение (16) примет вид

$$
v(x)=A_{2}(x)\left(D_{0+}^{1-\alpha-\beta} \tau(t)\right)(x)+B_{2}(x)\left(D_{1-}^{1-\alpha-\beta} \tau(t)\right)(x)+D_{2}(x) \tau(x),
$$

где

$$
\begin{gathered}
A_{2}(x)=-\frac{\Gamma(\alpha+\beta)}{\Gamma(\beta)} \cdot \frac{A(x)}{E_{2}(x)}, \quad B_{2}(x)=-\frac{\Gamma(\alpha+\beta)}{\Gamma(\alpha)} \cdot \frac{B(x)}{E_{2}(x)}, \quad D_{2}(x)=-\frac{D(x)}{E_{2}(x)}, \\
E_{2}(x)=\Gamma(2-\alpha-\beta)\left(\frac{m+2}{4}\right)^{\frac{2}{m+2}}\left[\frac{A(x)}{\Gamma(1-\alpha)}+\frac{B(x)}{\Gamma(1-\beta)}\right]+C(x),
\end{gathered}
$$

$\left(D_{0+}^{1-\alpha-\beta} f\right)(x)$ и $\left(D_{1-}^{1-\alpha-\beta} f\right)(x)$ - операторы дробного дифференцирования в смысле Римана-Лиувилля [2, с. 44], [7, с. 9]. Отсюда

$$
\begin{aligned}
& I^{*}=\int_{0}^{1} \tau(x) v(x) d x=\frac{1}{\Gamma(1-\alpha-\beta)} \int_{0}^{1} A_{2}(x) \tau(x) d x\left[\frac{d}{d x} \int_{0}^{x} \frac{\tau(t) d t}{(x-t)^{1-\alpha-\beta}}\right]- \\
& -\frac{1}{\Gamma(1-\alpha-\beta)} \int_{0}^{1} B_{2}(x) \tau(x) d x\left[\frac{d}{d x} \int_{x}^{1} \frac{\tau(t) d t}{(t-x)^{1-\alpha-\beta}}\right]+\int_{0}^{1} D_{2}(x) \tau^{2}(x) d x .
\end{aligned}
$$

Введём обозначения

$$
\begin{aligned}
& \frac{\sin ((\alpha+\beta) \pi)}{\pi} \frac{d}{d x} \int_{0}^{x} \frac{\tau(t) d t}{(x-t)^{1-\alpha-\beta}}=\tau_{1}(x), \\
& -\frac{\sin ((\alpha+\beta) \pi)}{\pi} \frac{d}{d x} \int_{x}^{1} \frac{\tau(t) d t}{(t-x)^{1-\alpha-\beta}}=\tau_{2}(x)
\end{aligned}
$$


и применим формулу обращения интегрального уравнения Абеля. Получим соответственно

$$
\tau(x)=\int_{0}^{x} \frac{\tau_{1}(t) d t}{(x-t)^{\alpha+\beta}}, \quad \tau(x)=\int_{x}^{1} \frac{\tau_{2}(t) d t}{(t-x)^{\alpha+\beta}} .
$$

Отсюда

$$
\begin{aligned}
& \frac{\Gamma(1-\alpha-\beta) \pi}{\sin ((\alpha+\beta) \pi)} I^{*}=\int_{0}^{1} A_{2}(x) \tau_{1}(x) d x \int_{0}^{x} \frac{\tau_{1}(\xi) d \xi}{(x-\xi)^{\alpha+\beta}}+ \\
& \quad+\int_{0}^{1} B_{2}(x) \tau_{2}(x) d x \int_{x}^{1} \frac{\tau_{2}(\xi) d \xi}{(\xi-x)^{\alpha+\beta}}+\frac{\Gamma(1-\alpha-\beta) \pi}{\sin ((\alpha+\beta) \pi)} \int_{0}^{1} D_{2}(x) \tau^{2}(x) d x .
\end{aligned}
$$

Воспользуемся известной формулой

$$
\int_{0}^{\infty} t^{\mu-1} \cos (k t) d t=\frac{\Gamma(\mu)}{k^{\mu}} \cos \left(\frac{\mu \pi}{2}\right) \quad(k>0,0<\mu<1) .
$$

Полагая $k=|x-\xi|, \mu=\alpha+\beta$, получим

$$
\frac{1}{|x-\xi|^{\alpha+\beta}}=\frac{1}{\Gamma(\alpha+\beta) \cos \left(\frac{\pi(\alpha+\beta)}{2}\right)} \int_{0}^{\infty} t^{\alpha+\beta-1} \cos (t|x-\xi|) d t .
$$

Поменяв порядок интегрирования в (17), а затем интегрируя по частям, находим

$$
\begin{aligned}
& \mu I^{*}=\mu \int_{0}^{1} D_{2}(x) \tau^{2}(x) d x- \\
&-\frac{1}{2} \int_{0}^{\infty} t^{\alpha+\beta-1} d t \int_{0}^{1} A_{2}^{\prime}(x)[ {\left[\int_{0}^{x} \tau_{1}(\xi) \cos (t \xi) d \xi\right)^{2}+} \\
&\left.+\left(\int_{0}^{x} \tau_{1}(\xi) \sin (t \xi) d \xi\right)^{2}\right] d x+ \\
&+\frac{1}{2} \int_{0}^{\infty} t^{\alpha+\beta-1} d t \int_{0}^{1} B_{2}^{\prime}(x)\left[\left(\int_{x}^{1} \tau_{2}(\xi) \cos (t \xi) d \xi\right)^{2}+\right. \\
&\left.+\left(\int_{x}^{1} \tau_{2}(\xi) \sin (t \xi) d \xi\right)^{2}\right] d x
\end{aligned}
$$

где

$$
\mu=\left[\frac{\pi}{\sin ((\alpha+\beta) \pi)}\right]^{2} \cos \left(\frac{(\alpha+\beta) \pi)}{2}\right)>0 .
$$

Очевидно, что при выполнении условий (13) имеем $I^{*} \geqslant 0$.

Пусть теперь выполняются условия (8)-(10) теоремы. Докажем, что и в этом случае $I^{*} \geqslant 0$. Действительно, при $\gamma(x)=0$ соотношение (16) примет вид

$$
\tau(x)=A_{1}(x)\left(I_{0+}^{1-\alpha-\beta} v(t)\right)(x)+B_{1}(x)\left(I_{1-}^{1-\alpha-\beta} v(t)\right)(x)+C_{1}(x) v(x),
$$


где

$$
\begin{gathered}
A_{1}(x)=-\frac{\Gamma(2-\alpha-\beta)}{\Gamma(1-\alpha)}\left(\frac{m+2}{4}\right)^{\frac{2}{m+2}} \frac{A(x)}{E_{1}(x)}, \\
B_{1}(x)=-\frac{\Gamma(2-\alpha-\beta)}{\Gamma(1-\beta)}\left(\frac{m+2}{4}\right)^{\frac{2}{m+2}} \frac{B(x)}{E_{1}(x)}, \\
C_{1}(x)=-\frac{C(x)}{E_{1}(x)}, \quad E_{1}(x)=\frac{\Gamma(\alpha+\beta)}{\Gamma(\beta)} A(x)+\frac{\Gamma(\alpha+\beta)}{\Gamma(\alpha)} B(x)+D(x),
\end{gathered}
$$

$\left(I_{0+}^{1-\alpha-\beta} f\right)(x)$ и $\left(I_{1-}^{1-\alpha-\beta} f\right)(x)$ - операторы дробного интегрирования в смысле Римана-Лиувилля [2, с. 42], [7, с. 9]. Отсюда

$$
\begin{aligned}
I^{*}= & \frac{1}{\Gamma(1-\alpha-\beta)} \int_{0}^{1} A_{1}(x) v(x) d x \int_{0}^{x} \frac{v(t) d t}{(x-t)^{\alpha+\beta}}+ \\
& +\frac{1}{\Gamma(1-\alpha-\beta)} \int_{0}^{1} B_{1}(x) v(x) d x \int_{x}^{1} \frac{v(t) d t}{(t-x)^{\alpha+\beta}}+\int_{0}^{1} C_{1}(x) v^{2}(x) d x .
\end{aligned}
$$

Проведя аналогичные преобразования, как мы делали выше, получим

$$
\begin{aligned}
\frac{\pi}{\sin \left(\pi \frac{\alpha+\beta}{2}\right)} I^{*}= & \frac{\pi}{\sin \left(\pi \frac{\alpha+\beta}{2}\right)} \int_{0}^{1} C_{1}(x) v^{2}(x) d x- \\
- & \int_{0}^{\infty} t^{\alpha+\beta-1} d t \int_{0}^{1} A_{1}^{\prime}(x)\left[\left(\int_{0}^{x} v(\xi) \cos (t \xi) d \xi\right)^{2}+\right. \\
& \left.\quad+\left(\int_{0}^{x} v(\xi) \sin (t \xi) d \xi\right)^{2}\right] d x+ \\
+ & \int_{0}^{\infty} t^{\alpha+\beta-1} d t \int_{0}^{1} B_{1}^{\prime}(x)\left[\left(\int_{x}^{1} v(\xi) \cos (t \xi) d \xi\right)^{2}+\right. \\
& \left.\quad+\left(\int_{x}^{1} v(\xi) \sin (t \xi) d \xi\right)^{2}\right] d x .
\end{aligned}
$$

Учитывая условия (10) теоремы и принимая во внимание, что

$$
\alpha+\beta=\frac{m}{m+2}, \quad \frac{\pi}{4} \leqslant \frac{\alpha+\beta}{2} \pi<\frac{\pi}{2}, \quad \sin \left(\frac{\alpha+\beta}{2} \pi\right)>0,
$$

из $(20)$ получаем оценку $I^{*} \geqslant 0$.

Таким образом, окончательно имеем $I^{*}=0$. Теперь заметим, что если в (20) $C_{1}(x) \neq 0$, сразу же следует $v(x)=0$. Если же $\left(A_{1}^{\prime}(x)\right)^{2}+\left(B_{1}^{\prime}(x)\right)^{2} \neq 0$, то поскольку $t^{\alpha+\beta-1}>0$, можно заключить, что

$$
\int_{0}^{x} v(\xi) \cos (t \xi) d \xi=0, \quad \int_{0}^{x} v(\xi) \sin (t \xi) d \xi=0
$$

или

$$
\int_{x}^{1} v(\xi) \cos (t \xi) d \xi=0, \quad \int_{x}^{1} v(\xi) \sin (t \xi) d \xi=0
$$

30 
для всех $t \in[0, \infty)$, в частности при $t=2 \pi k, k=0,1,2, \ldots$.

При этих значениях $t$ функции $\sin (t \xi), \cos (t \xi)$ образуют полную ортогональную систему функций в $L^{2}$. Следовательно, $v(\xi)=0$ почти всюду, а так как $v(x)$ непрерывна по условию, то $v(\xi)=0$ всюду. Отсюда $v(x)=0$ и из (19) при $\gamma(x)=0$ следует, что $\tau(x)=0$. Подобные рассуждения справедливы, если $I^{*}$ записан и в форме $(18)$. Следовательно, $u(x, y) \equiv 0$ в области $\Omega_{2}$ как решение задачи Коши для уравнения (1) с нулевыми данными, а в области $\Omega_{1}$ $u(x, y) \equiv 0$ как решение однородной задачи (1)-(3) при выполнении условия (5) теоремы, доказанной в работе [9].

2. Существование решения задачи. Соотношение между $\tau(x)$ и $v(x)$, принесенное на $\bar{I}$ из области $\Omega_{1}$, имеет вид

$$
\tau^{\prime \prime \prime}(x)+a_{1}(x) \tau^{\prime}(x)+a_{0}(x) \tau(x)-b(x) v(x)=0 .
$$

Проинтегрируем (21) трижды от 0 до $x$. После вычислений, учитывая, что $u(0, y)=\varphi_{1}(y)$, получим [5]

$$
\begin{aligned}
\tau(x)+\int_{0}^{x}\left[(x-\xi) a_{1}(\xi, 0)\right. & \left.+\frac{1}{2}(x-\xi)^{2}\left[a_{0}(\xi, 0)-a_{1}^{\prime}(\xi, 0)\right]\right] \tau(\xi) d \xi- \\
- & \frac{1}{2} \int_{0}^{x}(x-\xi)^{2} b(\xi, 0) v(\xi) d \xi= \\
= & {\left[1+a_{1}(0,0) \frac{x^{2}}{2}\right] \varphi_{1}(0)+\tau^{\prime}(0)(x)+\tau^{\prime \prime}(0) \frac{x^{2}}{2} }
\end{aligned}
$$

Соотношение между $\tau(x)$ и $v(x)$ при выполнении условий (8), (9) теоремы и соотношения (19) имеет вид

$$
\begin{aligned}
\tau(x)=A_{1}(x)\left(I_{0+}^{1-\alpha-\beta} v(t)\right)(x)+B_{1}(x)\left(I_{1-}^{1-\alpha-\beta} v(t)\right) & (x)+ \\
& +C_{1}(x) v(x)+\gamma_{1}(x),
\end{aligned}
$$

где $\gamma_{1}(x)=\gamma(x) / E_{1}(x)$.

Подставляя $\tau(x)$ из $(23)$ в $(22)$, получим

$$
\begin{gathered}
A_{1}(x)\left(I_{0+}^{1-\alpha-\beta} v(t)\right)(x)+B_{1}(x)\left(I_{1-}^{1-\alpha-\beta} v(t)\right)(x)+C_{1}(x) v(x)- \\
-\frac{1}{2} \int_{0}^{x}(x-\xi)^{2} b(\xi, 0) v(\xi) d \xi+\int_{0}^{x} M(x, \xi)\left[\frac{A_{1}(\xi)}{\Gamma(1-\alpha-\beta)} \times\right. \\
\left.\times \int_{0}^{\xi} \frac{v(t) d t}{(\xi-t)^{\alpha+\beta}}+\frac{B_{1}(\xi)}{\Gamma(1-\alpha-\beta)} \int_{\xi}^{1} \frac{v(t) d t}{(t-\xi)^{\alpha+\beta}}+C_{1}(\xi) v(\xi)\right] d \xi=f(x),
\end{gathered}
$$

где

$$
\begin{gathered}
M(x, \xi)=(x-\xi) a_{1}(\xi, 0)+\frac{1}{2}(x-\xi)^{2}\left[a_{0}(\xi, 0)-a_{1}^{\prime}(\xi, 0)\right] \\
f(x)=\left[1+a_{1}(0,0) \frac{x^{2}}{2}\right] \varphi_{1}(0)+\tau^{\prime}(0) x+\tau^{\prime \prime}(0) \frac{x^{2}}{2}-\gamma_{1}(x)-\int_{0}^{x} M(x, \xi) \gamma_{1}(x) d \xi
\end{gathered}
$$


Поменяем порядок интегрирования в двойных интегралах и при условии $C_{1}(x) \neq 0 x \in \bar{I}$ перепишем уравнение (24) в виде

$$
v(x)+\int_{0}^{1} k(x, \xi) v(\xi) d \xi=F(x)
$$

где

$$
\begin{gathered}
F(x)=\frac{f(x)}{C_{1}(x)}, \quad k(x, \xi)= \begin{cases}k_{1}(x, \xi), & \xi \leqslant x, \\
k_{2}(x, \xi), & \xi \geqslant x,\end{cases} \\
k_{1}(x, \xi)=\frac{1}{C_{1}(x) \Gamma(1-\alpha-\beta)}\left[\frac{A_{1}(x)}{(x-\xi)^{\alpha+\beta}}-\frac{1}{2} \Gamma(1-\alpha-\beta)(x-\xi)^{2} b(\xi, 0)+\right. \\
\left.+\int_{\xi}^{x} \frac{M(x, t) A_{1}(t) d t}{(t-\xi)^{\alpha+\beta}}+\int_{0}^{\xi} \frac{M(x, t) B_{1}(t) d t}{(\xi-t)^{\alpha+\beta}}+\Gamma(1-\alpha-\beta) M(x, \xi) C_{1}(\xi)\right], \\
k_{2}(x, \xi)=\frac{1}{C_{1}(x) \Gamma(1-\alpha-\beta)}\left[\frac{B_{1}(x)}{(\xi-x)^{\alpha+\beta}}+\right. \\
\left.+\int_{0}^{x} \frac{M(x, t) B_{1}(t) d t}{(\xi-t)^{\alpha+\beta}}+\Gamma(1-\alpha-\beta) M(x, \xi) C_{1}(\xi)\right] .
\end{gathered}
$$

Таким образом, ядро уравнения $(25) k(x, \xi) \in C(\bar{I} \times \bar{I}) \cap C^{1}(I \times I)$ при $\xi \neq x$, а при $\xi=x$ допускает оценку

$$
k(x, \xi)=\frac{k^{*}(x, \xi)}{|x-\xi|^{\alpha+\beta}}=\frac{0(1)}{|x-\xi|^{\alpha+\beta}}
$$

и уравнение (25) - это уравнение Фредгольма второго рода, безусловная разрешимость которого следует из единственности решения задачи. Единственное решение уравнения (25) находится по формуле [10]

$$
\tau(x)=F(x)+\int_{0}^{1} R(x, t) F(t) d t
$$

где $R(x, t)$ - резольвента ядра $K(x, t)$.

Пусть теперь выполняются условия (11), (12) теоремы единственности. Тогда соотношение между $\tau(x)$ и $v(x)$ из области $\Omega_{2}$ имеет вид

$$
\begin{aligned}
v(x)=A_{2}(x)\left(D_{0+}^{1-\alpha-\beta} \tau(t)\right)(x)+B_{2}(x)\left(D_{1-}^{1-\alpha-\beta} \tau(t)\right)(x)+ \\
+ \\
+D_{2}(x) \tau(x)+\gamma_{2}(x),
\end{aligned}
$$

где $\gamma_{2}(x)=\gamma(x) / E_{2}(x)$.

Подставив (26) в (22), будем иметь 


$$
\begin{gathered}
\tau(x)+\int_{0}^{\xi} M(x, \xi) \tau(\xi) d \xi- \\
-\frac{1}{2} \int_{0}^{x} \frac{(x-\xi)^{2} b(\xi, 0)}{\Gamma(\alpha+\beta)}\left[A_{2}(\xi) \frac{d}{d \xi} \int_{0}^{\xi} \frac{\tau(t) d t}{(\xi-t)^{\alpha+\beta}}-B_{2}(\xi) \frac{d}{d \xi} \int_{\xi}^{1} \frac{\tau(t) d t}{(t-\xi)^{\alpha+\beta}}\right] d \xi- \\
-\frac{1}{2} \int_{0}^{x}(x-\xi)^{2} b(\xi, 0) D_{2}(\xi) \tau(\xi) d \xi=\frac{1}{2} \int_{0}^{x}(x-\xi)^{2} b(\xi, 0) \gamma_{2}(\xi) d \xi+ \\
+\left[1+a_{1}(0,0) \frac{x^{2}}{2}\right] \varphi_{1}(0)+\tau^{\prime}(0) x+\tau^{\prime \prime}(0) \frac{x^{2}}{2} .
\end{gathered}
$$

Преобразуем интегральное уравнение (27). Для этого рассмотрим

$$
I_{1}(x)=\int_{0}^{x}(x-\xi)^{2} b(\xi, 0) A_{2}(\xi)\left[\frac{d}{d \xi} \int_{0}^{\xi} \frac{\tau(t) d t}{(\xi-t)^{\alpha+\beta}}\right] d \xi .
$$

Интегрируя по частям, а затем, меняя порядок интегрирования, получим

$$
I_{1}(x)=-\int_{0}^{x} \tau(t) d t \int_{t}^{x} \frac{\left[(x-\xi)^{2} b(\xi, 0) A_{2}(\xi)\right]_{\xi}^{\prime}}{(\xi-t)^{\alpha+\beta}} d \xi .
$$

Аналогично

$$
\begin{aligned}
& I_{2}(x)=\int_{0}^{x}(x-\xi)^{2} b(\xi, 0) B_{2}(\xi) {\left[\frac{d}{d \xi} \int_{\xi}^{1} \frac{\tau(t) d t}{(t-\xi)^{\alpha+\beta}}\right] d \xi=} \\
&=-\int_{0}^{x} \tau(t) d t \int_{0}^{t} \frac{\left[(x-\xi)^{2} b(\xi, 0) B_{2}(\xi)\right]_{\xi}^{\prime}}{(t-\xi)^{\alpha+\beta}} d \xi- \\
& \quad-\int_{x}^{1} \tau(t) d t \int_{0}^{x} \frac{\left[(x-\xi)^{2} b(\xi, 0) B_{2}(\xi)\right]_{\xi}^{\prime}}{(t-\xi)^{\alpha+\beta}} d \xi .
\end{aligned}
$$

На основании представлений $I_{1}(x)$ и $I_{2}(x)$ уравнение $(27)$ можно переписать следующим образом:

$$
\tau(x)+\int_{0}^{1} N(x, t) \tau(t) d t=F_{1}(x),
$$

где

$$
\begin{gathered}
N(x, t)= \begin{cases}N_{1}(x, t), & t \leqslant x \\
N_{2}(x, t), & t \geqslant x\end{cases} \\
N_{1}(x, t)=(x-t) a_{1}(t, 0)+\frac{1}{2}(x-t)^{2}\left[a_{0}(t, 0)-a_{1}^{\prime}(t, 0)\right]+ \\
+\frac{1}{2 \Gamma(\alpha+\beta)}\left(\int_{t}^{x}\left[(x-\xi)^{2} b(\xi, 0) A_{2}(\xi)\right]_{\xi}^{\prime} \frac{d \xi}{(\xi-t)^{\alpha+\beta}}-\right. \\
\left.-\int_{0}^{t}\left[(x-\xi)^{2} b(\xi, 0) B_{2}(\xi)\right]_{\xi}^{\prime} \frac{d \xi}{(t-\xi)^{\alpha+\beta}}\right)-\frac{1}{2}(x-t)^{2} b(t, 0) D_{2}(t),
\end{gathered}
$$




$$
\begin{gathered}
N_{2}(x, t)=-\frac{1}{2 \Gamma(\alpha+\beta)} \int_{0}^{x}\left[(x-\xi)^{2} b(\xi, 0) B_{2}(\xi)\right]_{\xi}^{\prime} \frac{d \xi}{(t-\xi)^{\alpha+\beta}}, \\
F_{1}(x)=\frac{1}{2} \int_{0}^{x}(x-\xi)^{2} b(\xi, 0) \gamma_{2}(\xi) d \xi+\left[1+a_{1}(0,0) \frac{x^{2}}{2}\right] \varphi_{1}(0)+\tau^{\prime}(0) x+\tau^{\prime \prime}(0) \frac{x^{2}}{2} .
\end{gathered}
$$

В силу сделанных ранее предположений относительно гладкости известных функций можно заключить, что $N(x, t) \in C(\bar{I} \times \bar{I}) \cap C^{3}(I \times I), F_{1}(x) \in$ $C^{1}(\bar{I}) \cap C^{3}(I)$.

Таким образом, уравнение (28) есть уравнение Фредгольма второго рода относительно $\tau(x)$, безусловная разрешимость которого в требуемом классе функций следует из единственности решения задачи. Единственное решение уравнения (28) может быть найдено по формуле

$$
\tau(x)=F_{1}(x)+\int_{0}^{1} R(x, t) F_{1}(t) d t
$$

где $R(x, t)$ - резольвента ядра $N(x, t)$.

Неизвестные постоянные $\tau^{\prime}(0)$ и $\tau^{\prime \prime}(0)$, входящие в $F_{1}(x)$, определим из (27), пользуясь краевыми условиями (2), (3), из которых следует, что

$$
\tau(1)=\varphi_{2}(0), \quad \tau^{\prime}(0)-\tau^{\prime}(1)=\varphi_{3}(0) .
$$

Действительно,

$$
F_{1}(x)=\tau^{\prime}(0) x+\tau^{\prime \prime}(0) \frac{x^{2}}{2}+g(x)
$$

где

$$
g(x)=\left[1+a_{1}(0,0) \frac{x^{2}}{2}\right] \varphi_{1}(0)+\frac{1}{2} \int_{0}^{x}(x-\xi)^{2} b(\xi, 0) \gamma_{2}(\xi) d \xi .
$$

Отсюда

$$
\begin{gathered}
F_{1}^{\prime}(x)=\tau^{\prime}(0)+\tau^{\prime \prime}(0) x+g^{\prime}(x) \\
g^{\prime}(x)=a_{1}(0,0) \varphi_{1}(0) x+\int_{0}^{x}(x-\xi) b(\xi, 0) \gamma_{2}(\xi) d \xi .
\end{gathered}
$$

Легко видеть, что

$$
\begin{gathered}
g(0)=\varphi_{1}(0), \quad g(1)=\left[1+\frac{a_{1}(0,0)}{2}\right] \varphi_{1}(0)+\frac{1}{2} \int_{0}^{1}(1-\xi)^{2} b(\xi, 0) \gamma_{2}(\xi) d \xi \\
g^{\prime}(0)=0, \quad g^{\prime}(1)=a_{1}(0,0) \varphi_{1}(0)+\int_{0}^{1}(1-\xi) b(\xi, 0) \gamma_{2}(\xi) d \xi
\end{gathered}
$$

Так как $\tau(1)=\varphi_{2}(0)$, то из $(29)$ и (30) имеем

$$
\varphi_{2}(0)=\tau^{\prime}(0)+\frac{\tau^{\prime \prime}(0)}{2}+g(1)+\int_{0}^{1} R(1, t)\left[\tau^{\prime}(0) t+\tau^{\prime \prime}(0) \frac{t^{2}}{2}+g(t)\right] d t
$$

или, что тоже самое, 


$$
\begin{aligned}
&\left(1+\int_{0}^{1} R(1, t) t d t\right) \tau^{\prime}(0)+\frac{1}{2}\left(1+\int_{0}^{1} R(1, t) t^{2} d t\right) \tau^{\prime \prime}(0)= \\
&=\varphi_{2}(0)-g(1)-\int_{0}^{1} R(1, t) g(t) d t .
\end{aligned}
$$

Далее заметим, что

$$
\begin{gathered}
\tau^{\prime}(x)=\tau^{\prime}(0)+\tau^{\prime \prime}(0) x+g^{\prime}(x)+\int_{0}^{1} R_{x}^{\prime}(x, t)\left[\tau^{\prime}(0) t+\tau^{\prime \prime}(0) \frac{t^{2}}{2}+g(t)\right] d t, \\
\tau_{1}^{\prime}(0)-\tau^{\prime}(1)=\varphi_{3}(0) \quad \text { и } \quad g^{\prime}(0)=0,
\end{gathered}
$$

а также

$$
\begin{array}{r}
\tau^{\prime}(0) \int_{0}^{1}\left[R_{x}^{\prime}(0, t)-R_{x}^{\prime}(1, t)\right] t d t+\frac{\tau^{\prime \prime}(0)}{2}\left(\int_{0}^{1}\left[R_{x}^{\prime}(0, t)-R_{x}^{\prime}(1, t)\right] t^{2} d t-1\right)= \\
=\varphi_{3}(0)+g^{\prime}(1)-\int_{0}^{1}\left[R_{x}^{\prime}(0, t)-R_{x}^{\prime}(1, t)\right] g(t) d t .
\end{array}
$$

Итак, получена система $(31),(32)$, из которой можно определить $\tau^{\prime}(0)$ и $\tau^{\prime \prime}(0)$. Система (31), (32) будет иметь единственное решение, если отличен от нуля определитель

$$
\left|\begin{array}{cc}
1+\int_{0}^{1} R(1, t) t d t & \frac{1}{2}\left(1+\int_{0}^{1} R(1, t) t^{2} d t\right) \\
\int_{0}^{1}\left[R_{x}^{\prime}(0, t)-R_{x}^{\prime}(1, t)\right] t d t & \frac{1}{2}\left(\int_{0}^{1}\left[R_{x}^{\prime}(0, t)-R_{x}^{\prime}(1, t)\right] t^{2} d t-1\right)
\end{array}\right| \neq 0
$$

Это завершает доказательство существования решения исследуемой задачи.

\section{БИБЛИОГРАФИЧЕСКИЙ СПИСОК}

1. Saigo M. A remark on integral operators involving the Gauss hypergeometric function // Math. Rep. Kyushu Univ, 1978. Vol.11, no. 2. Pp. 135-143.

2. Самко С.Г., Килбас А.А., Маричев О.И. Интегралы и производные дробного порядка и некоторые их приложения. Минск: Наука и техника, 1987. 688 с. [Samko S. G., Kilbas A.A., Marichev O.I. Integrals and derivatives of fractional order and some of their applications. Minsk: Nauka i Tekhnika, 1987. 688 pp.]

3. Репин О.А. Краевые задачи со смещением для уравнений гиперболического и смешанного типов. Самара: Изд-во Саратов. ун-та, Самарский филиал, 1992. 164 с. [Repin O.A. Boundary value problems with shift for equations of hyperbolic and mixed type. Samara: Izd-vo Saratov. Un-ta, Samarskiy Filial, 1992. 164 pp.]

4. Елеев B.A., Кумыкова C.К. Об одной краевой задаче со смещением для смешанного уравнения третьего порядка с кратными характеристиками / В сб.: Труды Всероссийской научной конференции. Часть 3: Дифференциальные уравнения и краевые задачи / Матем. моделирование и краев. задачи. Самара: СамГТУ, 2004. С. 91-94. [Eleev V.A., Kumykova S.K. On some boundary value problems with a shift for mixed third order equation with multiple characteristics / In: Proceedings of the All-Russian Scientific Conference (26-28 May 2004). Part 3 / Matem. Mod. Kraev. Zadachi. Samara: SamGTU, 2004. Pp. 91-94]. 
5. Елеев B. А., Кумыкова C. К. Внутреннекраевая задача для уравнения смешанного типа третьего порядка с кратными характеристиками // Известия Кабардино-Балкарского научного чентра PAH, 2010. №5(37), Часть 2. С. 5-14. [Eleev V. A., Kumykova S. K. The inner boundary value problem for mixed-type equation of third order with multiple characteristics // Izvestiya Kabardino-Balkarskogo nauchnogo tsentra RAN, 2010. no. 5(37), Part 2. Pp. 5-14].

6. Смирнов M. М. Вырождающиеся эллиптические и гиперболические уравнения. М.: Наука, 1966. 292 с. [Smirnov M. M. Degnerating elliptic and hyperbolic equations. Moscow: Nauka, 1966. 292 pp.]

7. Нахушев А. М. Дробное исчисление и его применение. М.: Физматлит, 2003. 271 с. [Nakhushev A. M. Fractional calculus and its applications. Moscow: Fizmatlit, 2003. 271 pp.]

8. Трикоми Ф. Лекции по уравнениям в частных производных. М.: Иностр. лит., 1957. 443 c. [Tricomi F. Lectures on partial differential equations. Moscow: Inostr. Lit., 1957. 443 pp.]

9. Иргашев Ю. Некоторые краевые задачи для уравнений третьего порядка с кратными характеристиками / В сб.: Краевые задачи для дифференциалъных уравнений и их приложения. Ташкент: Фан, 1976. C. 17-27. [Irgashev Yu. Some boundary value problems for third order equations with multiple characteristics / In: Boundary value problems for differential equations and their applications. Tashkent: Fan, 1976. Pp. 17-27].

10. Трикоми Ф. Интегральные уравнения. М.: Иностр. лит, 1960. 299 с. [Tricomi F. Integral equations. Moscow: Inostr. Lit., 1960. 299 pp.]

Поступила в редакцию $17 / \mathrm{X} / 2011$;

в окончательном варианте - 23/XI/2011.

MSC: 35M12; 26A33, 33C05

\section{NONLOCAL PROBLEM FOR A EQUATION OF MIXED TYPE OF THIRD ORDER WITH GENERALIZED OPERATORS OF FRACTIONAL INTEGRO-DIFFERENTIATION OF ARBITRARY ORDER}

\section{O.A. Repin ${ }^{1}$, S. K. Kumykova ${ }^{2}$}

1 Samara State Economic University,

141, Sovetskoy Armii st., Samara, 443090, Russia.

2 Kabardino-Balkarian State University,

173, Chernyshevskogo st., Nalchik, 360004, Russia.

E-mails: matstat@mail.ru, bsk@rect.kbsu.ru

The unique solvability of internally boundary value problem for equation of mixed type of third order with multiple characteristics is investigated. The uniqueness theorem is proved with the restrictions on certain features and different orders of fractional integro-differentiation. The existence of solution is equivalent reduced to a Fredholm integral equation of the second kind.

Key words: boundary value problem, fractional integro-differentiation operators, Gauss hypergeometric function, Fredholm integral equation.

Original article submitted $17 / \mathrm{X} / 2011$; revision submitted $23 / \mathrm{XI} / 2011$.

Oleg A. Repin (Dr. Sci. (Phys. \& Math.)), Head of Dept., Dept. of Mathematical Statistics \& Econometrics. Svetlana K. Kumykova (Ph. D. (Phys. \& Math.)), Associate Professor, Dept. of Function Theory. 